\title{
Spectroscopic analysis of the gaseous argon scintillation with a wavelength sensitive particle detector
}

\author{
R. Santorelli ${ }^{1, \mathrm{a}}{ }^{\infty}$, E. Sanchez Garcia ${ }^{1, \mathrm{~b}}$, P. Garcia Abia ${ }^{1}$ D. González-Díaz $^{2}$, R. Lopez Manzano ${ }^{1}$, \\ J. J. Martinez Morales ${ }^{1}$, V. Pesudo ${ }^{1}$, L. Romero ${ }^{1}$ \\ ${ }^{1}$ CIEMAT, Division de Física de Particulas, Avda. Complutense, 40, Madrid, Spain \\ ${ }^{2}$ IGFAE, Campus Vida, Rúa Xosé María Suárez Núñez, s/n, Santiago de Compostela, Spain
}

Received: 2 January 2021 / Accepted: 25 June 2021 / Published online: 17 July 2021

(C) The Author(s) 2021, corrected publication 2021

\begin{abstract}
We performed a time-resolved spectroscopic study of the VUV/UV scintillation of gaseous argon as a function of pressure and electric field, by means of a wavelength sensitive detector operated with different radioactive sources. Our work conveys new evidence of distinctive features of the argon light which are in contrast with the general assumption that, for particle detection purposes, the scintillation can be considered to be largely monochromatic at $128 \mathrm{~nm}$ (second continuum). The wavelength and time-resolved analysis of the photon emission reveal that the dominant component of the argon scintillation during the first tens of $n s$ is in the range $[160,325] \mathrm{nm}$. This light is consistent with the third continuum emission from highly charged argon ions/molecules. This component of the scintillation is field-independent up to $25 \mathrm{~V} / \mathrm{cm} / \mathrm{bar}$ and shows a very mild dependence with pressure in the range $[1,16]$ bar. The dynamics of the second continuum emission is dominated by the excimer formation time, whose variation as a function of pressure has been measured. Additionally, the time and pressure-dependent features of electron-ion recombination, in the second continuum band, have been measured. This study opens new paths toward a novel particle identification technique based on the spectral information of the noble-elements scintillation light.
\end{abstract}

\section{Introduction}

Over the last decade, argon and xenon detectors have attracted a lot of interest for their use in direct dark matter search [1-5] and neutrino experiments [6-8], given their unique ionization and scintillation properties. These experiments use high-purity noble elements as both interaction target and tracking medium for particles, attaining overall

\footnotetext{
a e-mail: r.santorelli@ ciemat.es (corresponding author)

b e-mail: edgar.sanchez@ ciemat.es (corresponding author)
}

performances better than the ones reachable with other technologies. In gas phase, their exceptional calorimetric properties are used for instance in $[9,10]$.

A central aspect of either single or dual phase (gas-liquid) noble element detectors is their efficient collection and detection of the vacuum ultraviolet (VUV) scintillation photons, which provides calorimetric data, event time for the $3 \mathrm{D}$ event reconstruction, and particle identification capability [11,12]. Experimental information, obtained largely from the closely related fields of photo-chemistry, plasma and laser physics, allows attributing this characteristic scintillation to the bond created between excited and ground state atoms through 3body collisions (see, e.g., [13]). The resulting singlet and triplet excimer states (Rydberg states with a dimer core and a binding electron) undergo radiative de-excitation, giving rise to the so-called second excimer continuum. It is typically assumed that this feature dominates the scintillation spectrum for gas pressures above 100 mbar, and results in relatively narrow emission bands $(\approx 10 \mathrm{~nm}$ wide) at $128 \mathrm{~nm}$ (argon) and $172 \mathrm{~nm}$ (xenon) [10,14].

Under the above paradigm, there has been so far little motivation towards exploiting spectroscopic information in this kind of particle detectors. As a consequence, the light detection systems of argon and xenon chambers are based on broad-band optical sensors, possibly coupled to photon wavelength-shifters, that effectively integrate the light signal over a wide spectral range, missing the potential information provided by the scintillation wavelength.

Indeed, studies were carried out (mostly prior to the 00's) to investigate the temporal evolution of characteristic spectral components of argon and xenon scintillation with strong Xray [15], electron [16-18], proton [19] or heavy ion [20,21] beams. Although a high intensity beam is a simple way to produce the photon yields required to study scintillation with high spectroscopic detail, it is known to potentially introduce spurious effects such as heating, volume recombination, 
interactions with walls and emission of debris from the window (a comparison between the scintillation of an electron beam and a $\beta$-source can be found, for instance, in Fig. 9 of [22]). In contrast to the above studies, systematic investigations of the spectral and temporal characteristics and absolute scintillation yields under individual particles is much scarcer, and seems to be almost entirely circumscribed to $\alpha$-particles [23-25].

We built a high-pressure gas detector in the CIEMAT laboratory (Sect. 3) as part of a project for the spectroscopic study of the argon and xenon scintillation with different radioactive sources. The use of photon sensors with different spectral sensitivities allowed us to detect the Ar scintillation in the near, middle and far UV ranges, and to investigate, in detail, aspects of the photon production mechanism and light emission spectrum particularly relevant for particle detectors based on noble elements.

Results obtained with $\alpha$ and $\beta$ sources deployed in the gas chamber, operated up to 20 bar, evidenced a substantial emission in the middle-UV region, which is consistent with the so-called third continuum emission (Sects. 2 and 4). Despite being still sub-dominant, overall, compared to the second continuum, the third continuum is markedly fast, hence representing the main contribution to the photon signal during the first tens of ns (Sects. 4 and 5), for pressures below 10 bar.

To the best of our knowledge, this is the first systematic study of the third continuum carried out with $\alpha$ and $\beta$ sources operated in a noble-gas chamber. Our findings significantly boost the importance of the spectral information in noble-element detectors. Although the two continua can be unambiguously differentiated spectroscopically, the widespread use of wavelength shifter coatings in dark matter and neutrino detectors spoils any possibility of exploiting the spectroscopic information of the argon scintillation. The demonstration of a light emission mechanism more complex than the one so far considered in the literature, with well separated emission bands for different particle types, could trigger the interest in a novel detector concept sensitive to the photon wavelength. This technology would allow to exploit distinctive features of the scintillation of noble elements, a possibility currently not conceived by the present experiments.

\section{Brief review of the scintillation mechanisms in noble gases}

In this section we revisit briefly the most accepted model describing the mechanisms through which scintillation light is generated, starting with the 1st and 2nd continuum. The emission originates from vibrationally-relaxed singlet $\left(\operatorname{Ar}_{2}^{*}\left({ }^{1} \Sigma_{u}\right)\right)$ and triplet $\left(\operatorname{Ar}_{2}^{*}\left({ }^{3} \Sigma_{u}\right)\right)$ excimer states, when tran- sitioning to the dissociative ground state of the Ar dimer $\left(\operatorname{Ar}_{2}\left({ }^{1} \Sigma_{g}\right)\right)$. The importance of this emission stems from the fact that, for pressures above few 100's of mbar, low-lying excited atomic states (resonant: $\operatorname{Ar}^{*}\left(s_{4}\right)$, and metastable: $\left.\mathrm{Ar}^{*}\left(s_{5}\right)\right)$ are quickly and predominantly populated through a collision-dominated atomic cascade; given that singlet and triplet excimer states are formed from those atomic states through termolecular (3-body) reactions, this emission displays a high universality. Focusing on the $\operatorname{Ar}^{*}\left(s_{4}\right)$ state, the scintillation process can be characterized in general through the following (dominant) pathways:

$$
\begin{aligned}
& \mathrm{Ar}^{* *}+\mathrm{Ar} \stackrel{\text { (cascade) }}{\longrightarrow} \mathrm{Ar}^{*}\left(s_{4}\right)+\mathrm{Ar} \\
& \text { collisional quenching } \\
& \mathrm{Ar}^{* *} \stackrel{\text { (cascade) }}{\longrightarrow} \mathrm{Ar}^{*}\left(s_{4}\right)+h \nu^{\prime} \mathrm{s}
\end{aligned}
$$

atomic radiation

$\operatorname{Ar}^{*}\left(s_{4}\right)+\mathrm{Ar}+\mathrm{Ar} \stackrel{K_{s_{4} \rightarrow 0_{u}}}{\longrightarrow} \operatorname{Ar}_{2}^{*}\left(0_{u}^{+}\right)+\mathrm{Ar}$

(weakly bound) excimer formation

$$
\begin{aligned}
& \operatorname{Ar}_{2}^{*}\left(0_{u}^{+}\right) \stackrel{K_{0_{u} \rightarrow s_{4}}}{\longrightarrow} \operatorname{Ar}^{*}\left(s_{4}\right)+\mathrm{Ar} \\
& \text { excimer dissociation to } s_{4} \\
& \operatorname{Ar}_{2}^{*}\left(0_{u}^{+}\right) \stackrel{K_{0_{u} \rightarrow s_{5}}}{\longrightarrow} \operatorname{Ar}^{*}\left(s_{5}\right)+\mathrm{Ar} \\
& \text { excimer dissociation to } s_{5} \\
& \operatorname{Ar}_{2}^{*}\left(0_{u}^{+}\right) \stackrel{1 / \tau_{0_{u}}}{\longrightarrow} \mathrm{Ar}+\mathrm{Ar}+h v \\
& \text { first continuum } \\
& \operatorname{Ar}_{2}^{*}\left(0_{u}^{+}\right)+\operatorname{Ar} \stackrel{K_{\text {bind }, 1}}{\longrightarrow} \operatorname{Ar}_{2}^{*}\left({ }^{1} \Sigma_{u}(v)\right)+\operatorname{Ar} \\
& \text { excimer binding }
\end{aligned}
$$

$$
\begin{aligned}
& \operatorname{Ar}_{2}^{*}\left({ }^{1} \Sigma_{u}(v=0)\right) \stackrel{1 / \tau_{e_{2}}^{s}}{\longrightarrow} \operatorname{Ar}_{2}\left({ }^{1} \Sigma_{g}\right)+\operatorname{Ar}+h v \\
& \text { second continuum }
\end{aligned}
$$

Here $\mathrm{Ar}^{* *}$ refers to any excited atomic state above the two lowest ones, $\operatorname{Ar}_{2}^{*}\left(0_{u}^{+}\right)$is the weakly bound $\mathrm{Ar}_{2}^{*}$ excimer associated with $\operatorname{Ar}_{2}^{*}\left({ }^{1} \Sigma_{u}(v)\right)$, in Hund's notation corresponding to case $c$ [26], and the quantum number $v$ refers to the vibrational state, with $v=0$ corresponding to the bottom of the 
potential well. If considering, instead, that the cascade proceeds through the $\operatorname{Ar}^{*}\left(s_{5}\right)$ state, the situation is analogous:

$$
\begin{gathered}
\mathrm{Ar}^{* *}+\operatorname{Ar} \underset{\text { collisional quenching }}{\stackrel{\text { (cascade) }}{\longrightarrow}} \operatorname{Ar}^{*}\left(s_{5}\right)+\mathrm{Ar} \\
\operatorname{Ar}^{* *} \stackrel{\text { (cascade) }}{\longrightarrow} \operatorname{Ar}^{*}\left(s_{5}\right)+h v^{\prime} \mathrm{s} \\
\text { atomic radiation } \\
\operatorname{Ar}^{*}\left(s_{5}\right)+\mathrm{Ar}+\mathrm{Ar} \stackrel{K_{s_{5} \rightarrow 0_{u}}}{\longrightarrow} \operatorname{Ar}_{2}^{*}\left(1_{u} / 0_{u}^{-}\right)+\mathrm{Ar}
\end{gathered}
$$

(weakly bound) excimer formation

$$
\begin{aligned}
& \operatorname{Ar}_{2}^{*}\left(1_{u} / 0_{u}^{-}\right) \stackrel{K_{0_{u} \rightarrow s_{5}}}{\longrightarrow} \operatorname{Ar}^{*}\left(s_{5}\right)+\mathrm{Ar} \\
& \text { dissociation to } s_{5} \\
& \operatorname{Ar}_{2}^{*}\left(1_{u} / 0_{u}^{-}\right) \stackrel{1 / \tau_{1_{u}}}{\longrightarrow} \mathrm{Ar}+\mathrm{Ar}+h v
\end{aligned}
$$

first continuum

$$
\operatorname{Ar}_{2}^{*}\left(1_{u} / 0_{u}^{-}\right)+\operatorname{Ar} \underset{\text { excimer binding }}{\stackrel{K_{\text {bind }, 3}}{\longrightarrow}} \operatorname{Ar}_{2}^{*}\left({ }^{3} \Sigma_{u}(v)\right)+\operatorname{Ar}
$$

$$
\operatorname{Ar}_{2}^{*}\left({ }^{3} \Sigma_{u}(v)\right)+\operatorname{Ar} \underset{\text { excimer cooling/relaxation }}{\stackrel{K_{c o o l}, 3}{\longrightarrow}} \operatorname{Ar}_{2}^{*}\left({ }^{3} \Sigma_{u}(v=0)\right)+\operatorname{Ar}
$$

$$
\operatorname{Ar}_{2}^{*}\left({ }^{3} \Sigma_{u}(v=0)\right) \stackrel{1 / \tau_{e_{2}}^{t}}{\longrightarrow} \operatorname{Ar}_{2}\left({ }^{1} \Sigma_{g}\right)+\mathrm{Ar}+h v
$$

second continuum

and $\operatorname{Ar}_{2}^{*}\left(1_{u} / 0_{u}^{-}\right)$refers to the degenerate weakly bound excimers associated with $\operatorname{Ar}_{2}^{*}\left({ }^{3} \Sigma_{u}(v)\right)$. A detailed diagram compiling these pathways can be found for instance in [22]. In this work, reaction rates $(K)$ are assumed to depend on the number density $(N)$ and therefore are expressed in units of $\left[\mathrm{t}^{-1}\right]$. Experimentally, it has been determined that, for pressures above 100 mbar, the dominant time constants are the (3-body) formation times $\tau_{f_{2}}=1 / K_{S_{5(4)} \rightarrow 0_{u}}$ (Eqs. 2, 7) and the singlet and triplet decay times $\tau_{e_{2}}^{s}, \tau_{e_{2}}^{t}$ (Eqs. 5, 10).

At high ionization densities, characteristic of high pressures and/or highly ionizing radiation, charge recombination provides additional scintillation channels $[27,28]$. From

$$
\begin{aligned}
\mathrm{Ar}^{+}+\mathrm{Ar}+\mathrm{Ar} & \rightarrow \mathrm{Ar}_{2}^{+}+\mathrm{Ar} \\
\mathrm{Ar}_{2}^{+}+e & \rightarrow \mathrm{Ar}_{2}^{* *} \\
\mathrm{Ar}_{2}^{* *} & \rightarrow \mathrm{Ar}^{* *}+\mathrm{Ar}
\end{aligned}
$$

the 1st and 2nd continua follow, according to reactions 1a, $1 \mathrm{~b}, 6 \mathrm{a}, 6 \mathrm{~b}$. This leads to a perfect correlation between recombined charge and excess scintillation, with reaction 12 being regulated by the external electric field [29]. As a result, the effective energy to create a UV photon at zero-field varies in argon, under $\alpha$-particles, from $W_{s c}=50.6 \mathrm{eV}$ at 2 bar to $W_{s c}=25.3 \mathrm{eV}$ at 10 bar [30].

Finally, there is a set of mechanisms leading to scintillation mainly in the region $[180,300] \mathrm{nm}$, that are grouped under the generic denomination of "3rd continuum". The identification of the precursors to this continuum has been subject of hot debates throughout the 1980's and 1990's [31,32]. It is possible that a number of different species contribute to this continuum. Ref. [21], which seems to be the most complete study to date, combines the two leading hypotheses: doubly ionized $\left(\mathrm{Ar}^{++}\right)$and excited $\left(\mathrm{Ar}^{+*}\right)$ ions, However, that work does not allow quantitative predictions of the scintillation yields at low pressure, nor has it been fully substantiated. In a nutshell, doubly ionized states would lead to scintillation around $200 \mathrm{~nm}$ through 3-body reactions, as:

$$
\begin{aligned}
\mathrm{Ar}^{++}+\mathrm{Ar}+\mathrm{Ar} & \rightarrow\left(\mathrm{Ar}_{2}\right)^{++}+\mathrm{Ar} \\
\left(\mathrm{Ar}_{2}\right)^{++} & \rightarrow \mathrm{Ar}^{+}+\mathrm{Ar}^{+}+h v
\end{aligned}
$$

At high pressures, the presence of new peaks in the range $[200,300] \mathrm{nm}$ led to the consideration of additional contributions, finding a good correspondence with the molecular ion $\left(\mathrm{Ar}_{3}^{++}, \mathrm{Ar}_{2}^{+*}\right.$ and $\left.\mathrm{Ar}_{3}^{+*}\right)$ emission. According to [32] they would decay following:

$$
\begin{aligned}
\mathrm{Ar}_{3}^{++} & \rightarrow \mathrm{Ar}^{+}+\mathrm{Ar}^{+}\left(\text {or } \mathrm{Ar}_{2}^{++}\right)+\mathrm{Ar}+h v \\
\mathrm{Ar}_{2}^{+*} & \rightarrow \mathrm{Ar}^{+}+\mathrm{Ar}+h v \\
\mathrm{Ar}_{3}^{+*} & \rightarrow \mathrm{Ar}^{+}+\mathrm{Ar}+\mathrm{Ar}+h v
\end{aligned}
$$

Contrary to the 1 st and 2 nd continua, the pathways leading to formation of $\mathrm{Ar}_{n}^{+*}$ and $\mathrm{Ar}_{n}^{++}$species have not been unambiguously identified. According to experimental data obtained in [23] for $\alpha$-particles, electron-ion recombination is not competing with process 14 , so the 3 rd continuum would be largely field-independent except perhaps for very highly ionizing radiation.

\section{Experimental setup}

The wavelength-sensitive chamber is shown in Fig. 1. It consists of a $7 \mathrm{~cm}$ side stainless steel cube with one CF-40 flange on each face. The top and bottom flanges are connected to the service lines for vacuum and gas filling. The lateral flanges are equipped with four custom-made optical view-ports necessary to decouple the pressurized region from the optical readout system (Fig. 2). A custom-designed steel frame keeps the windows in place. Grooves in the mechanical assembly hold viton o-rings which seal the window, making the viewport vacuum and pressure-tight up to $20 \mathrm{bar}$. The windows are made of $8.0 \mathrm{~mm}$ thick UV-grade $\mathrm{MgF}_{2}$ crystals $(38.1 \mathrm{~mm}-$ $\varnothing$ ), whose transmission is $\approx 95 \%$ for wavelengths above 


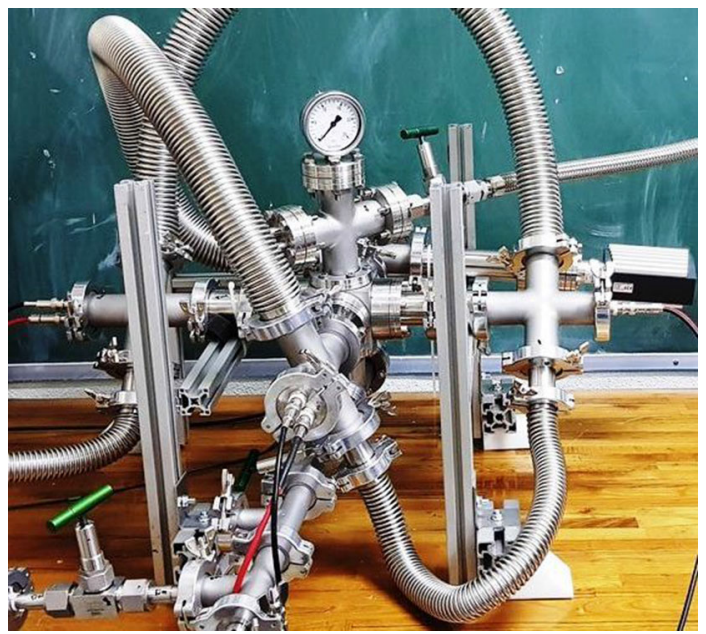

Fig. 1 Photograph of the experimental setup

$180 \mathrm{~nm}$ (Fig. 3). A transmission of $\approx 33 \%$ at $128 \mathrm{~nm}$ was measured by comparing the average signal of the argon slow component detected by a PMT with and without the $\mathrm{MgF}_{2}$ crystals installed in the optical view-port.

Four 1" Hamamatsu photomultipliers (PMT), with different spectral sensitivities, are hosted in canisters attached to the optical view-ports. They are built with KF-40 tees and cross fittings and equipped with a connection for the vacuum line, separated from the main pressure chamber, and two electrical feedthroughs for the HV and PMT signals. Two R7378A PMTs are placed in opposite sides of the central chamber. In the other two confronting faces, one R6095 PMT and one R6835 PMT are set. The R6095 PMT is coated with a $200 \mu \mathrm{g} / \mathrm{cm}^{2}$ layer of tetraphenyl butadiene (TPB), a wavelength shifter that converts the UV photons to $420 \mathrm{~nm}$ with approximately $100 \%$ efficiency [33]. The nominal quantum efficiencies $(\mathrm{QE})$ of these phototubes and the $\mathrm{MgF}_{2}$ window's transmission coefficient are plotted in Fig. 4 as a function of the photon wavelength [34,35].

Recent studies, not entirely unequivocally, show some dependence of the average TPB shifting efficiency on the wavelength of the incident photon [36]. In our analysis, we assume that the TPB conversion efficiency is flat in the VUVUV region, making the R6095 spectral sensitivity constant in the range of interest $([100,325] \mathrm{nm})$. The QE of this tube is considered equal to half of the nominal value at $420 \mathrm{~nm}$ (30\%) to take into account the $50 \%$ probability of backward emission of the TPB-converted photons. The CsI photocathode of the R6835 is solar blind, thus the $420 \mathrm{~nm}$ photons, diffused backwards by the TPB coating of the R6095 PMT placed in front of it, do not produce a signal.

Specific tests performed in our laboratory with the R6835 PMT and a blue LED proved that this phototube is not sensitive to the $420 \mathrm{~nm}$ light. The geometry of the setup reduces the optical cross-talk between the $420 \mathrm{~nm}$ photons and the

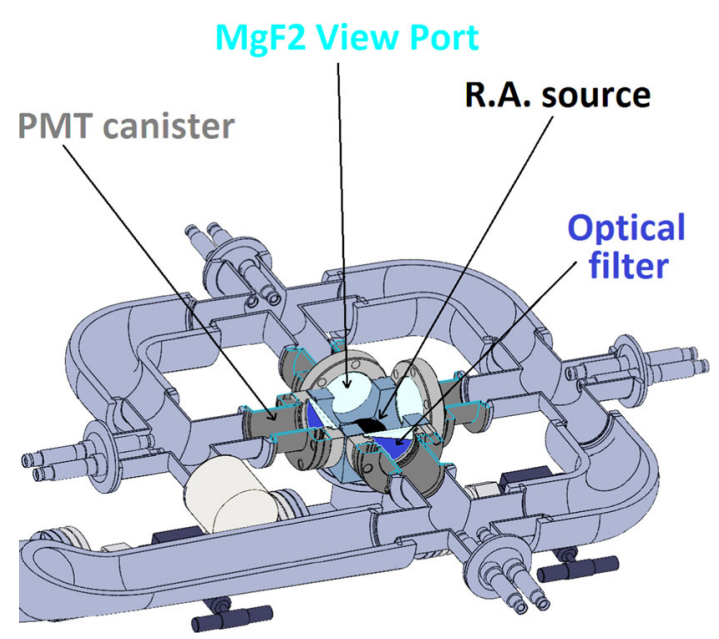

Fig. 2 Top view of detector. The pressurized region with the radioactive source is in the centre of the chamber

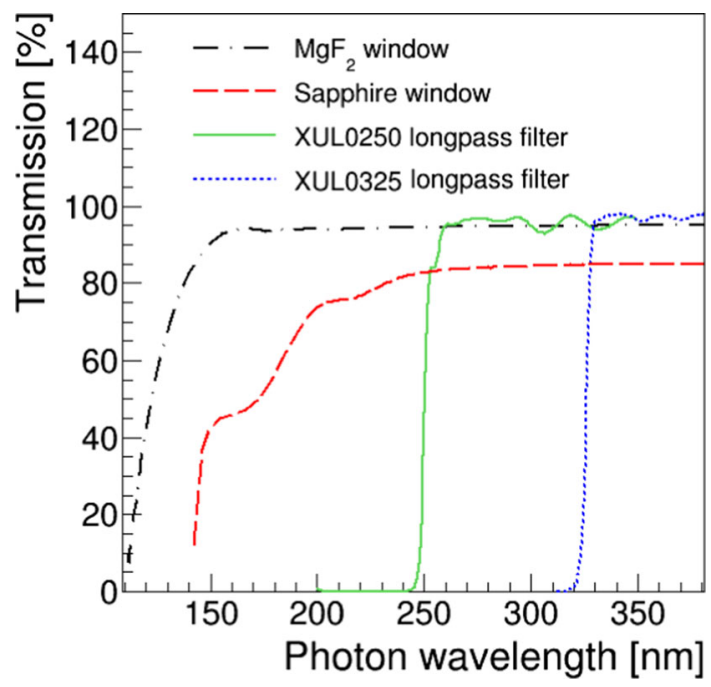

Fig. 3 Curves of light transmission as a function of wavelength, for the windows and filters used in the experiment

R7378 phototubes to a level which is negligible for our purposes.

Special runs with 1" $(\varnothing) \mathrm{Al}_{2} \mathrm{O}_{3}$ (Sapphire) crystals from Thorlabs [37] and long-pass filters from Asahi [38], placed in front of the R6835 and R7378, respectively, are also taken in order to narrow the spectral sensitivity of these phototubes. The cross-comparison of the signals with and without the filters placed in front of the PMTs allows to limit the detected Ar scintillation to well defined UV ranges. We constrain the argon scintillation light to the ranges of $[110,140] \mathrm{nm}$ (called UV2 region in the following) and [160, 325] nm (UV3 region) using the signals of the R6835 PMT with the $\mathrm{Al}_{2} \mathrm{O}_{3}$ window and of the $\mathrm{R} 7378$ PMT with the Asahi filters, respectively (Table 1). The upper limits are identified by the fact that no light signal is detected with the filters placed in front of the PMTs. 
Table 1 Definition of the UV2 and UV3 spectral ranges. The lower bounds are set by the spectral sensitivity of the PMTs. The upper limits are defined from the filters' transmission edge

\begin{tabular}{llll}
\hline Region & Range & Lower bound & Upper bound \\
\hline UV2 & {$[110,140] \mathrm{nm}$} & R6835 lower spectral sensitivity limit & $\mathrm{R}^{2} 835+\mathrm{Al}_{2} \mathrm{O}_{3} \mathrm{crystal}$ \\
$\mathrm{UV3}$ & {$[160,325] \mathrm{nm}$} & $\mathrm{R} 7378$ lower spectral sensitivity limit & $\mathrm{R} 7378+\mathrm{XUL0325}$ filter \\
\hline
\end{tabular}

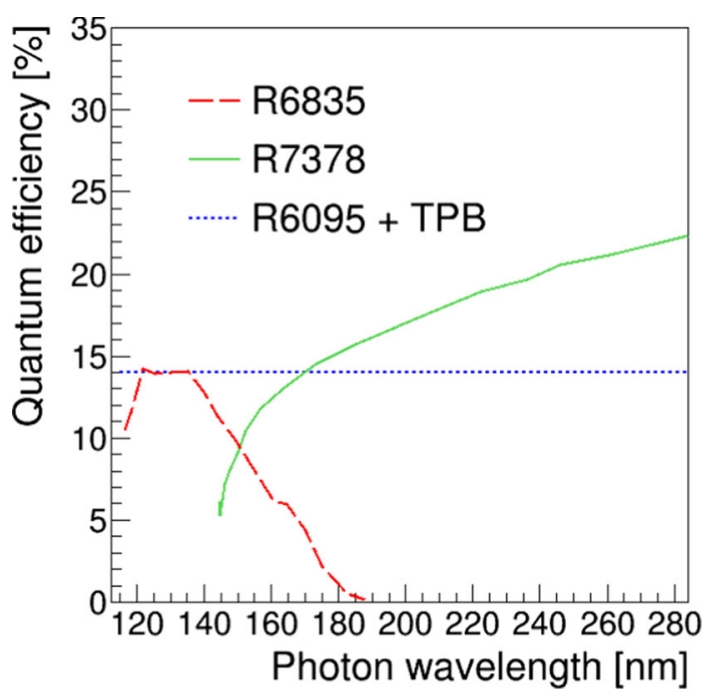

Fig. 4 Nominal quantum efficiencies of the PMTs used in the detector, measured by Hamamatsu as a function of the wavelength of the incident photons [34,35]

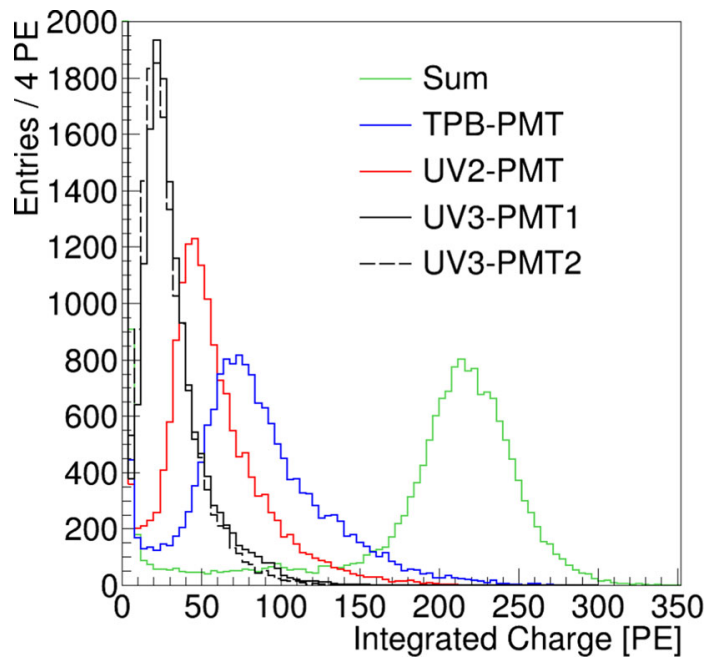

Fig. 5 Integrated charge signal of the four different PMTs used in the experiment, obtained with the ${ }^{241} \mathrm{Am} \alpha$ source in argon at 2 bar. The signal in the UV3 region is detected with two R7378 PMTs (UV3-PMT1 and UV3-PMT2). The light collected on the four photo detectors (Sum) is around $220 \mathrm{PE}$

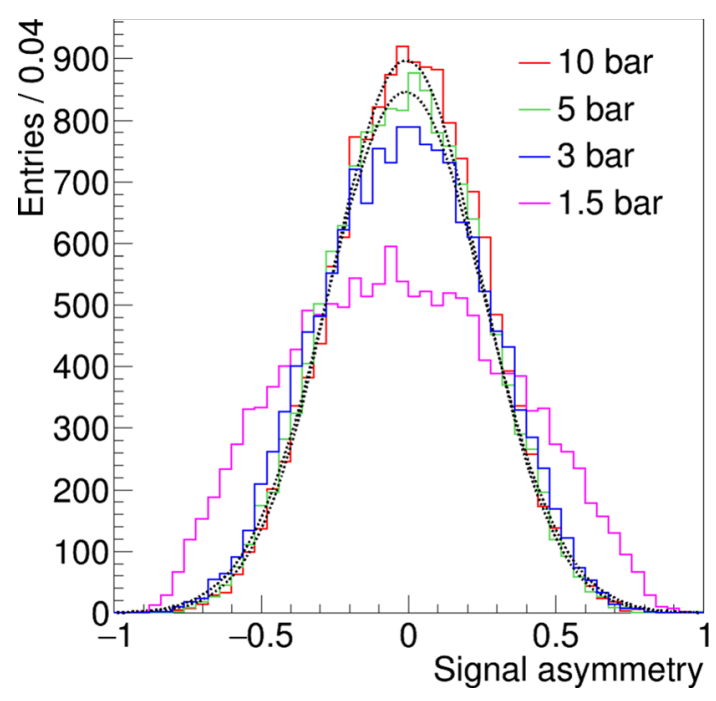

Fig. 6 Asymmetry between the two R7378 PMTs for different pressures. The Gaussian functions fitted to the data are overlaid

The detector is equipped with two pressure sensors (ITR90) and a set of $1 / 2^{\prime \prime}$ VCR valves that allow to set the pressure in the chamber and the optical readout system independently. The optical filters can be inserted or removed without the need to open or evacuate the pressure chamber. Before each run, the system is pumped with a TURBOVAC $350 \mathrm{i}$ for 24 hours and a pressure of $2 \times 10^{-5}$ mbar is typically reached in the central volume. Pure argon gas (AirLiquide ALPHAGAZTM $^{\mathrm{T}}$, purity $\geq 99.9999 \%$ ) is used to fill the chamber. The PMT region is evacuated and continuously pumped during the data taking (pressure $<9 \times 10^{-5}$ mbar) to minimize the absorption of the UV-VUV photons by the air between the optical view-port and the PMT window.

The PMTs are powered independently with a negative bias voltage through custom-made bleeder circuits with SMD components mounted on PCBs. The signal is amplified 10 times with a CAEN N979 module. One of the two outputs of the fast amplifier goes to a leading edge discriminator (CAEN N841), with a threshold set to 0.5 photoelectrons. Different trigger logics (CAEN N455 quad logic unit), based on the coincidence of the R6095 and R7378 phototubes, are used in the experiment. The second output of the fast amplifier is fed to a CAEN DT5725 sampling ADC (14-bit resolution and $250 \mathrm{MS} / \mathrm{s}$ sampling rate). Different runs are taken with digitization windows from 4 to $16 \mu \mathrm{s}$. The data are transferred 
via optical link to a computer for the analysis. The gain of the PMTs is obtained from dark-rate data taken in vacuum, by fitting the single photoelectron peak and the noise with a combination of Gaussian functions. The calibration of the R6835 is performed directly with the tail of the argon light pulse, given its very low dark pulse rate. The high voltage of the PMTs is set independently, in order to equalize the gains to the level of 500 ADC counts per photoelectron.

The gas purity is assessed through the decay time of the slow component of the argon second continuum emission. Simple selection criteria are applied to reject a few percent of the total triggers which are produced by electronic noise and cross-talk events.

The results presented in this study are obtained with ${ }^{241} \mathrm{Am}$ (activity $\approx 500 \mathrm{~Bq}$ ) and ${ }^{90} \mathrm{Sr} / \mathrm{Y}$ (activity $\left.\approx 100 \mathrm{~Bq}\right)$ radioactive sources electrodeposited on stainless steel disks. The pressure chamber is equipped with a PTFE support structure able to host one radioactive source in the centre.

\section{Wavelength-resolved UV/VUV emission with a ${ }^{241} \mathrm{Am}$ source}

Initial measurements were carried out with a ${ }^{241} \mathrm{Am} \alpha$ source in argon gas up to 16 bar using two R7378 (UV3PMT1 and UV3-PMT2 in the following), one R6835 (UV2PMT) and one TPB-coated R6095 PMT (TPB-PMT). Taking into account the $\mathrm{W}$-values reported in [30], $1.1 \times 10^{5}$ photons are produced in argon at 2 bar. Considering the solid angle between the PMTs photocathode and the source, the $\mathrm{MgF}_{2}$ transmission coefficient and the nominal PMTs QE, $(1.0 \pm 0.2) \times 10^{2}$ photoelectrons $(\mathrm{PE})$ are expected to be detected by the TPB-PMT, which is sensitive to the entire spectral range. The average solid angle between the PMTs photocathode and the source is estimated by means of a toy Monte Carlo which includes the detailed description of the chamber geometry and takes into account the refraction of the light on the $\mathrm{MgF}_{2}$ crystals. Geometrical efficiencies in the range [0.008, 0.011] are obtained for the different PMTs depending on the nominal photocathode size and the average length of the alpha track as a function of the gas pressure.

The $\alpha$-peak is clearly visible in the integrated charge spectra (Fig. 5). Depending on the gas purity and pressure, more than two hundreds of photoelectrons (PE) are detected in the full energy peak (green histogram) produced by the $\approx 5.5$ $\mathrm{MeV} \alpha$ particles, allowing wavelength and time-resolved analysis of the light pulses. The TPB-PMT detects $\approx 90 \mathrm{PE}$ on average (blue histogram in Fig. 5), a value which is in good agreement with the expected one. The UV2-PMT (red histogram) mean value is approximately $60 \mathrm{PE}$, while the two UV3-PMTs (black histograms), which are only sensitive to wavelengths above $160 \mathrm{~nm}$, detect $\approx 30 \mathrm{PE}$ each on average. This clearly establishes that there is a significant component of the argon scintillation light at wavelengths significantly longer than the $128 \mathrm{~nm}$ line from the second continuum.

The signal asymmetry, defined as the ratio between the difference and the sum of a pair of PMTs integrated signals, is plotted in Fig. 6 for the two UV3 phototubes at different pressure values. The data are fitted with a Gaussian function with a mean value compatible with zero, confirming that the source is at the centre of the chamber. The width of the distribution decreases with increasing pressure due to the reduction of the mean range of the particles in the gas, indicating that the influence of any geometrical correction in the measured scintillation is negligible above 3 bar.

The pulse shape of a typical signal, produced by an $\alpha$ event in argon at 1.5 bar, is presented in Fig. 7, as registered by the different PMTs. The TPB-PMT detects the characteristic fast and slow components of the argon emission. However, only the slow component of the scintillation is detected in the UV2 region, in form of a long train of pulses ( $\mu$ s scale) at the single photoelectron level. The UV3 sensitive phototubes detect only the prompt emission, with a pulse amplitude similar to the one detected by the TPB-PMT.

A signal consistent with dark current is registered by the UV2-PMT with the sapphire crystal placed between the PMT window and the $\mathrm{MgF}_{2}$ optical view-port, proving that the slow component of the Ar scintillation is entirely in the range [110, 140] nm. The light signal detected by the UV3-PMTs with the $250 \mathrm{~nm}$ long-pass filter is of the order of $20 \%$ of the total signal without filter. No light is detected with the $325 \mathrm{~nm}$ long-pass filter.

The 1.5 bar argon scintillation pulses, averaged over $3 \times 10^{4}$ events, are displayed in Fig. 8 in two time ranges. Only the $\alpha$-particle interactions from the ${ }^{241}$ Am source are considered, using an energy threshold cut. LED calibration runs taken in vacuum evidenced an after-pulsing component, between $60 \mathrm{~ns}$ and $100 \mathrm{~ns}$ after the maximum pulse height, in the UV3-PMTs signal. This second pulse is removed from the analysis with a software cut, after confirming that the impact in our study is negligible (Fig. 9).

In this analysis, the number of PEs as a function of time has been reported without any signal correction. The charge integral is corrected for the purity of the argon gas. This purity was estimated through the decay time constant $\tau_{e 2}^{t}$ of the triplet excimer states $\operatorname{Ar}_{2}^{*}\left({ }^{3} \Sigma_{u}\right)$. The values obtained between 2.6 and $2.8 \mu$ s set the level of impurities in the range 0.1-1 ppm [39], which is consistent with the gas contamination certified by the producer. The light yield via the triplet deexcitation is then scaled by $\tau_{e 2}^{t} / \tau_{\text {ref }}$, where $\tau_{e 2}^{t}$ is the measured triplet decay time and $\tau_{\text {ref }}=3.2 \mu \mathrm{s}$ is the triplet radiative lifetime with negligible impurities concentration [40]. The measured triplet decay time rejects the possible explanation of the UV3-photon production in terms of parasitic reemissions from $\mathrm{N}_{2}, \mathrm{H}_{2} \mathrm{O}$ and $\mathrm{O}_{2}$ contamination, which can only produce a sub-dominant component in the UV band and 

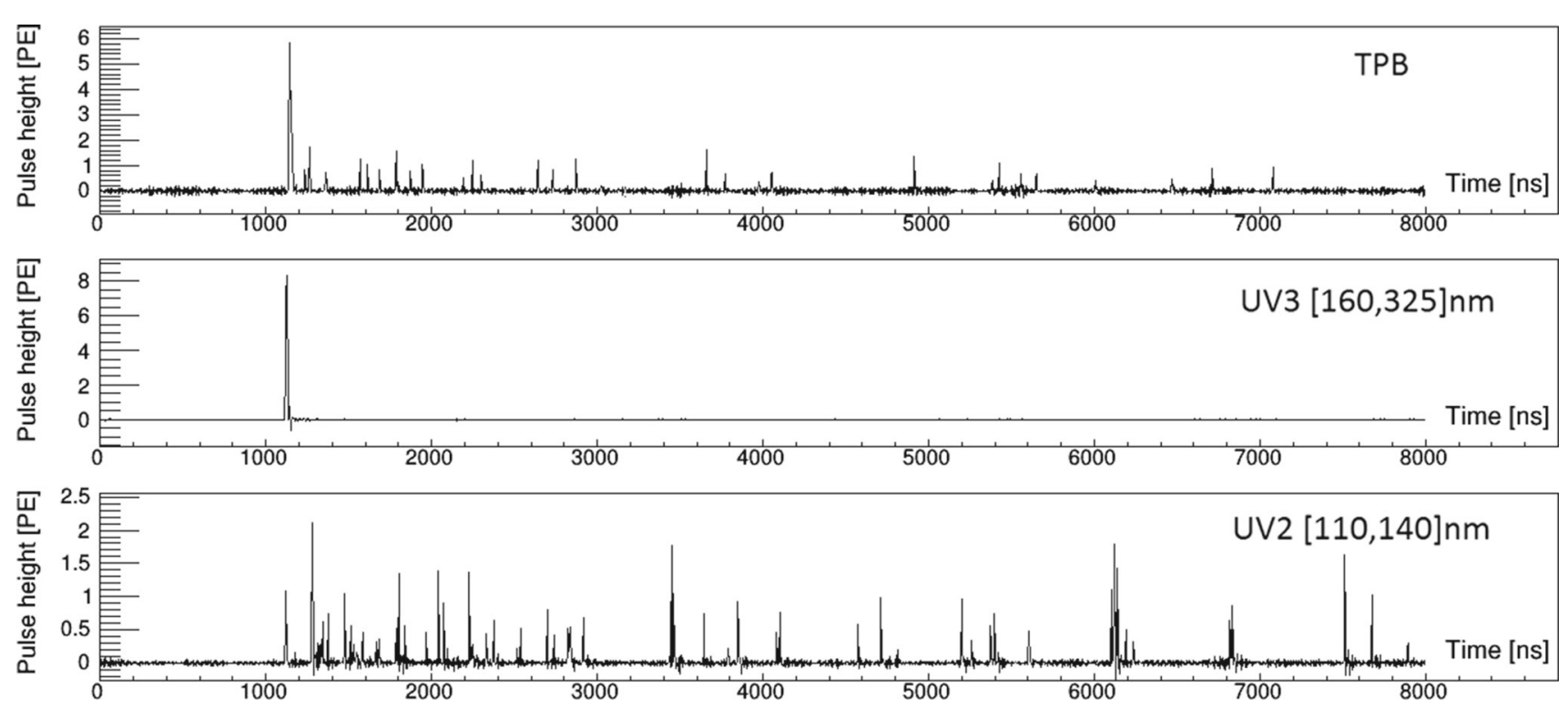

Fig. 7 Typical scintillation signals from the interaction of a $5.5 \mathrm{MeV} \alpha$-particle as detected in three spectral regions, for argon gas at 1.5 bar. A clear separation of the components is possible by wavelength discrimination

Fig. 8 Pulse shapes of the argon scintillation signals averaged over $3 \times 10^{4}$ events per PMT type, registered with the ${ }^{241} \mathrm{Am}$ source in Ar at 1.5 bar, in two time windows: (left) $[0,3000] \mathrm{ns}$ and (right) $[0,400]$ ns. The signal of only one PMT is considered for the histogram of the UV3 region
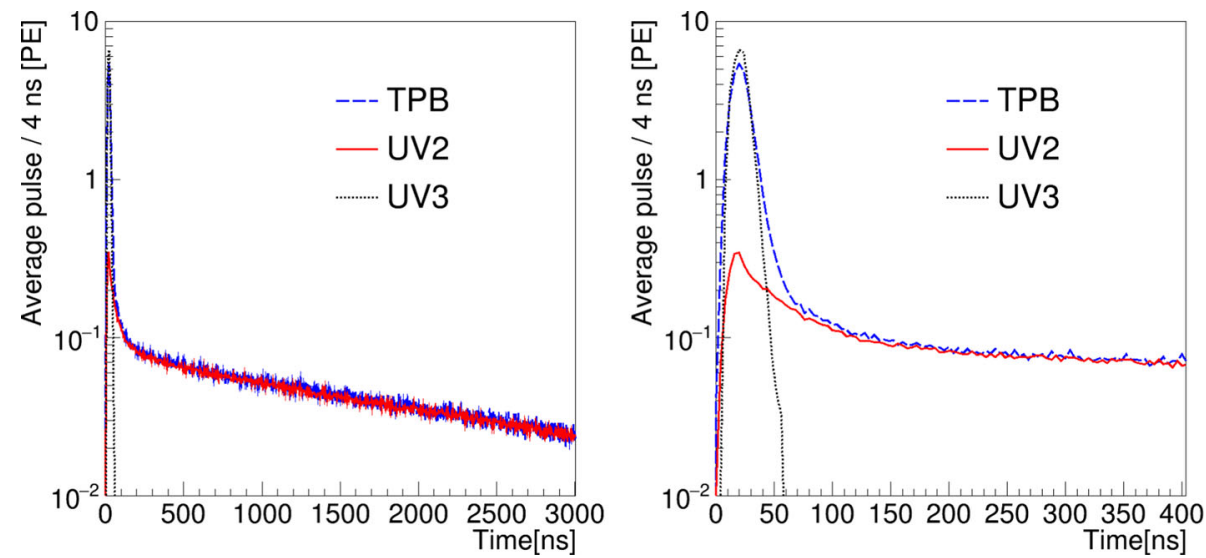

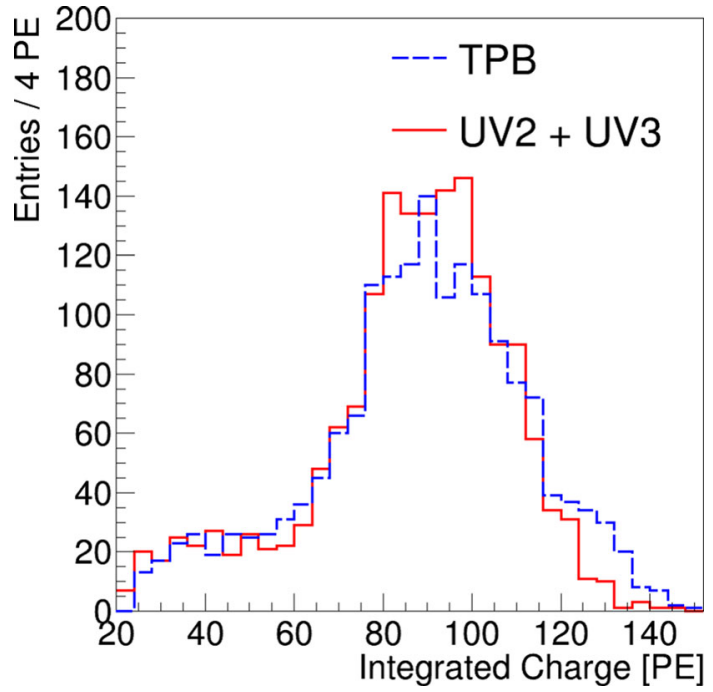

Fig. $9{ }^{241}$ Am data in argon gas at 1.5 bar: integrated charge spectrum obtained with the PMT covered with the wavelength shifter compared with the sum of the spectra of the UV2 and one of the UV3 PMT over a wide time scale. Any explanation, other than the third continuum emission, in terms of unusual Ar contaminants is considered implausible.

The combination of the plots in Figs. 5 and 8 suggests that the amplitude of the signal detected by the TPB-PMT, which is sensitive to the entire spectral range, is very similar to the sum of the signals of the UV2 and one of the UV3 PMTs. In Figs. 9 and 10, the integrated charge spectra and the average pulse shape of the argon scintillation are shown with the ${ }^{241} \mathrm{Am}$ source in argon gas at 1.5 bar, selecting the events in the center of the detector through two symmetry cuts between the different couples of confronting PMTs. Given the symmetry of the setup and the similar size of the PMTs, the fact that the tube signals are similar in the two regions UV2 and UV3 also suggests that the QEs are comparable.

These results prove that the two components of the scintillation with Ar gas at 1.5 bar are characterized by distinct wavelengths and can be unambiguously differentiated spectroscopically. The slow component is consistent with the sec- 


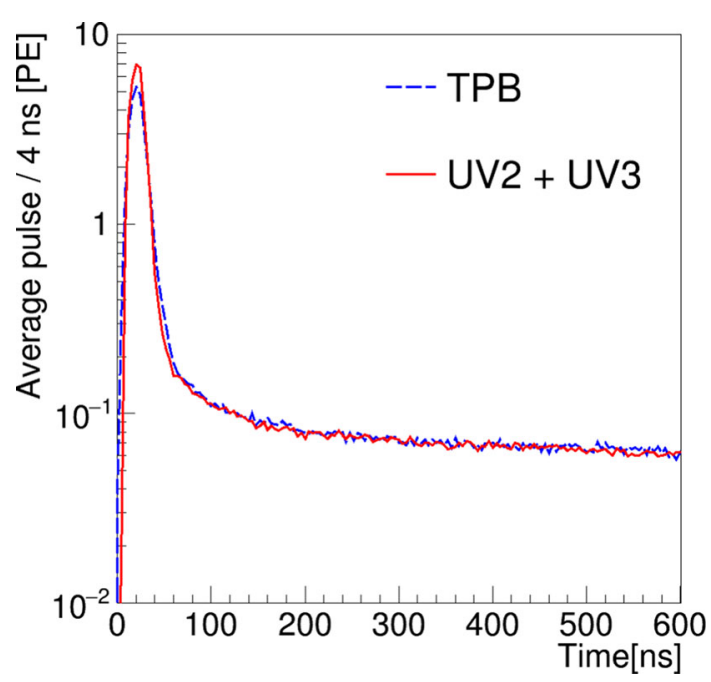

Fig. 10 Average argon pulse detected with the TPB and sum of the UV2 and UV3 pulses at 1.5 bar

ond continuum emission (Sect. 2), which gives rise to $128 \mathrm{~nm}$ photons. The fast component of the scintillation, on the other hand, is entirely in the UV3 region and it is compatible with the third continuum emission.

\section{Spectroscopic analysis of the argon scintillation as a function of gas pressure}

In order to quantitatively compare the mean scintillation signal in the two spectral regions UV2 and UV3, we calculate the total number of photons produced by the $\alpha$ interactions considering the $\mathrm{MgF}_{2}$ transmission values (33\% for the UV2 region and $95 \%$ for the UV3), the nominal QEs ( 0.15 for the UV2-PMT and TPB-PMT, 0.18 for the UV3-PMT) and the solid angle estimated through the toy Monte Carlo (Sect. 3). The analog manometer introduces an uncertainty of $0.2 \mathrm{bar}$ on the pressure measurement.

The fast/slow component ratio obtained with the TPBcoated PMT and 1.5 bar gas pressure is 5.4 , after corrections for the gas purity and the $\mathrm{MgF}_{2}$ window transmittance. This result is in good agreement with the value $5.5 \pm 0.6$ at 1.1 bar absolute pressure measured in [40].

The variation of the average ${ }^{241} \mathrm{Am}$ signal, defined as the mean value of a Gaussian fit to the $\alpha$ peak, is presented in Fig. 11 as a function of gas pressure.

The light yield of the chamber is sufficient to clearly identify the alpha peak in the charge spectra (Fig. 5), and the statistical uncertainty from the fit is negligible for all the PMTs. The fluctuations in the number of photons are taken into account with a pressure independent systematic uncertainty of $\pm 3-5 \%$, that is related to the uncertainty on the

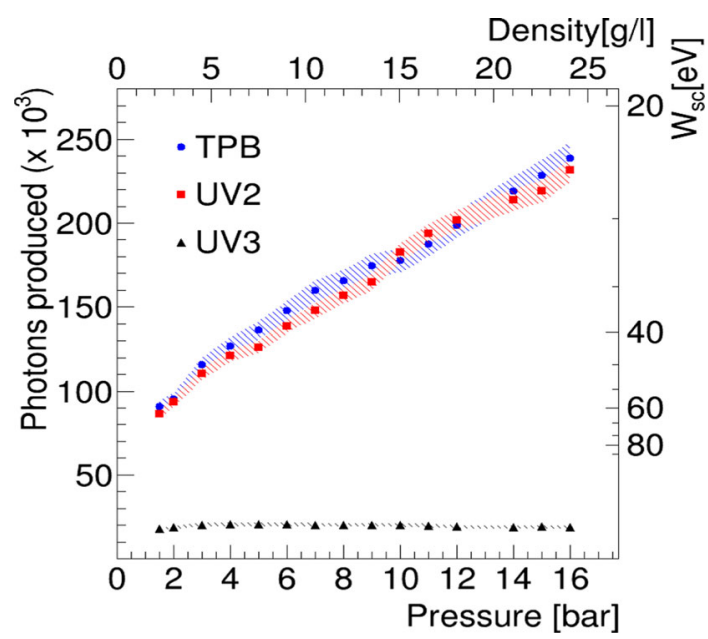

Fig. 11 Mean $\alpha$ signal detected in the different spectral ranges as a function of the pressure. The bands indicate the systematic uncertainties

gain calibration and to the different spectral responses of the PMTs.

The light in the UV2 region (red squares in Fig. 11) increases to more than twice the initial value in the $[1.5,16]$ bar pressure range, an effect which is consistent with the enhanced electron-ion recombination probability at higher gas pressure [25]. On the other hand, the signal in the UV3 region (black triangles) is stable within the uncertainties in that pressure range: this result suggests that the recombination light is consistent only with $128 \mathrm{~nm}$ photons production and no other emission is observed at longer wavelengths.

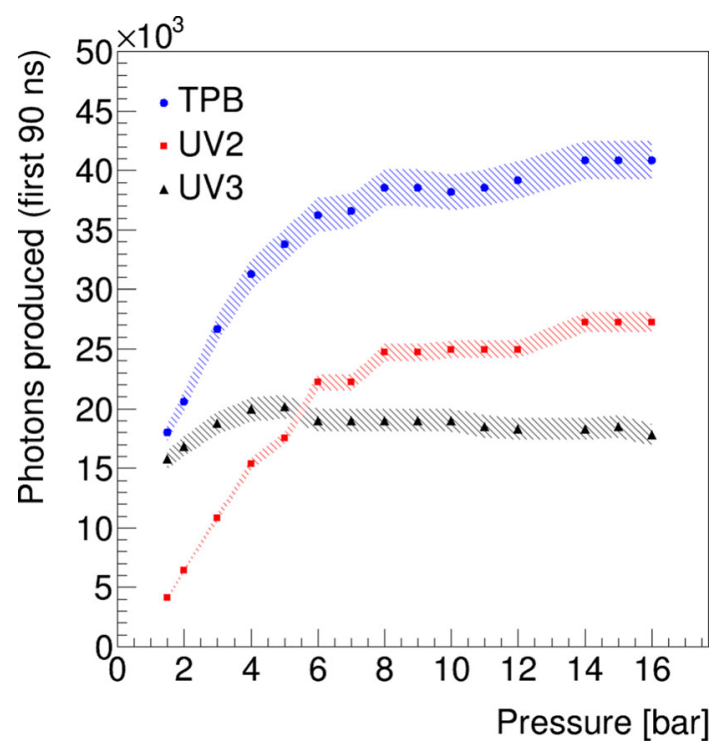

Fig. 12 Variation with the pressure of the fast component (first $90 \mathrm{~ns}$ ) of the argon scintillation. The bands indicate the systematic uncertainties 

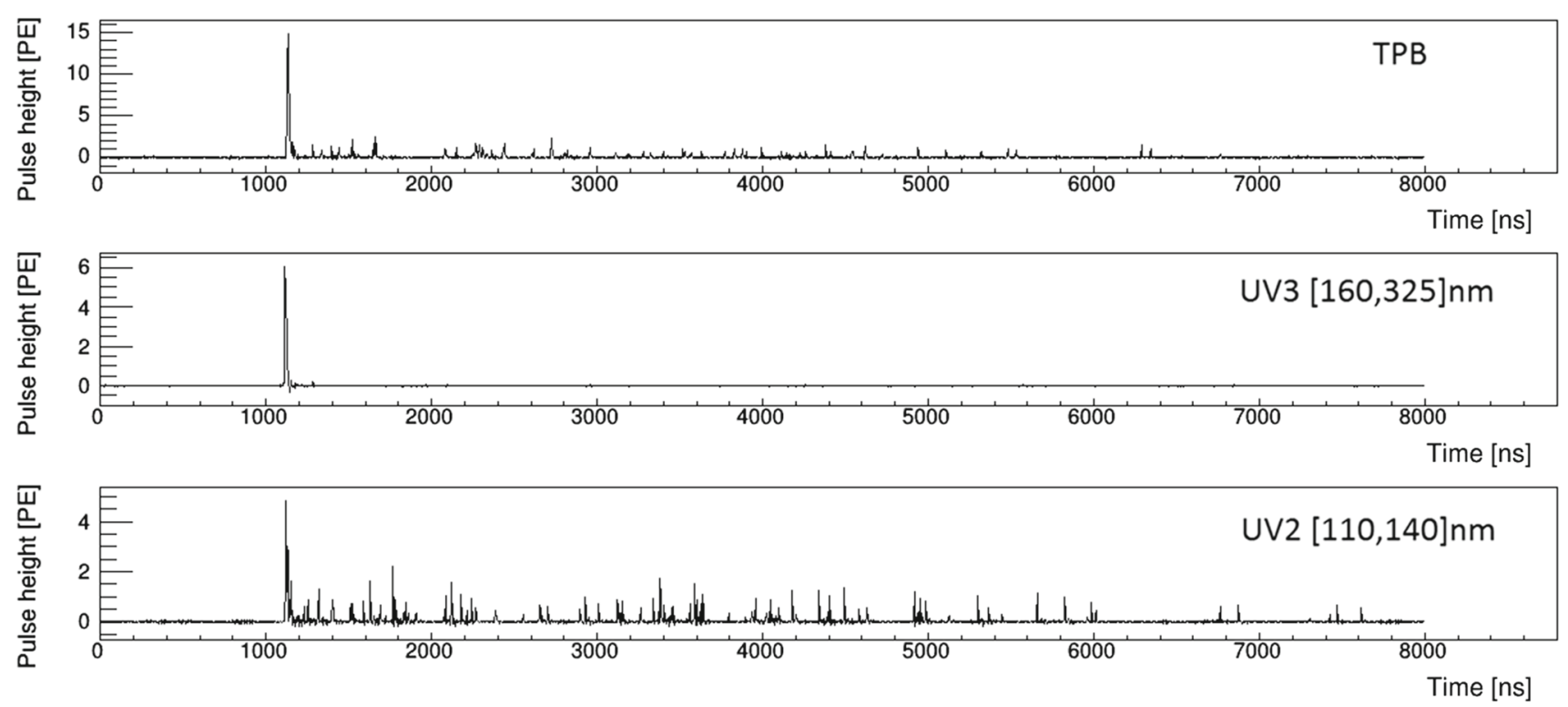

Fig. 13 Typical Ar scintillation signals from 5.5 MeV $\alpha$-particle interaction detected in three spectral regions, for argon gas at 16 bar. Compared to the typical signal at $1.5 \mathrm{bar}$ (Fig. 7), a fast component appears in the UV2 region when the pressure is increased

Fig. 14 Average signals in the $[1.5,16]$ bar pressure range for the (left) UV2 and (right) UV3 regions. Same normalization factor applied to all the curves shown in a single plot. For UV3, only slight variations on the shapes are observed for different pressures. In the UV2 region, the fast component is more significant at higher pressure
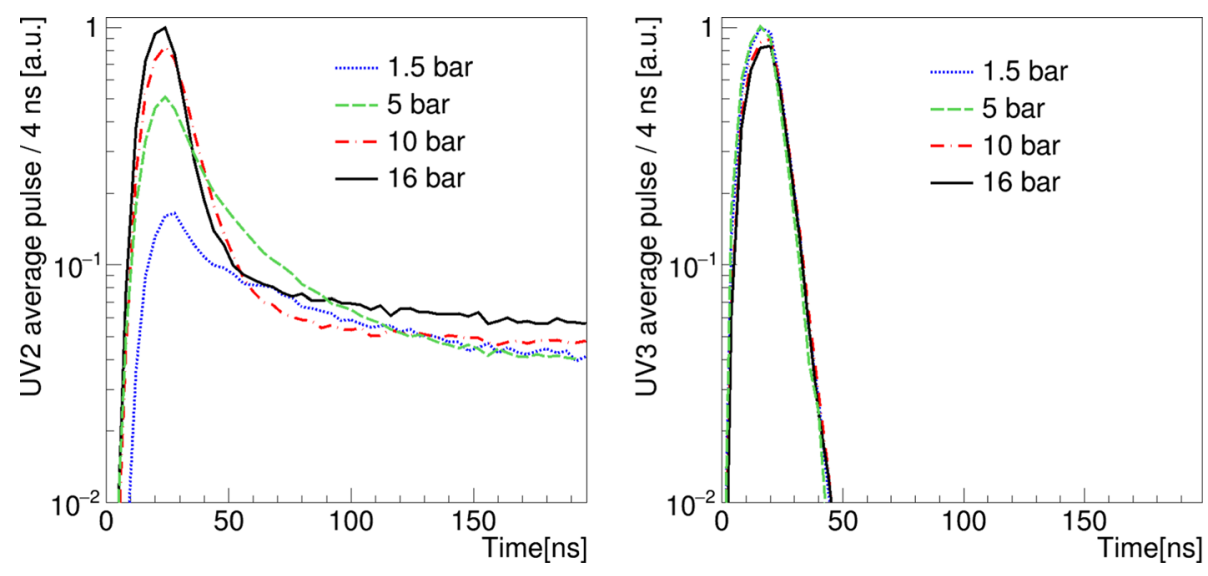

In Fig. 12 the evolution of the charge signal in a fixed $90 \mathrm{~ns}$ time window after the trigger ("fast" component) is shown as a function of the gas pressure. The UV2 signal rises up to $25 \mathrm{PE}$ and gets nearly stable at pressures above 7 bar, demonstrating that more photons are emitted promptly in the UV2 region (red dots) when the gas pressure increases. The UV3 component (black dots) barely depends on the pressure and represents the dominant light emission during the first $90 \mathrm{~ns}$, up to 5 bar.

A typical signal from an $\alpha$ interaction in argon at 16 bar is plotted in Fig. 13. When it is compared with the signal at 1.5 bar (Fig. 7), a fast component can be now observed in the UV2 region. In the UV3 region, though, the pulse shape and the amplitude of the signal is similar to the 1.5 bar case. This is consistent with the fast component of the scintillation signal detected by the TPB-PMT being significantly larger at 16 bar than at 1.5 bar.

The argon scintillation pulse, averaged over $3 \times 10^{4} \alpha$ interactions, is depicted in Fig. 14 for different pressures and spectral regions. The pulse heights are normalized to the maximum values, which are observed at 1.5 bar for the UV3 and 16 bar for the UV2 emissions, respectively. A relatively small decrease of the UV3 signal is evident as the pressure increases from 5 to 16 bar (Fig. 14-right). The enhancement of the fast argon scintillation component at higher pressure is evident in the UV2 region (Fig. 14-left). This effect is consistent with the reduction of the average distance among the argon molecules at higher pressure, which allows the formation of the excimer in shorter time and leads to an increase in the fast component through the decay of the singlet ${ }^{1} \sum_{u}$ states. This result is in agreement with an old study that evidenced the dependence of the $\mathrm{Ar}_{2}^{*}$-excimer formation-time on the gas pressure [41]. The change of slope around 6 bar in Fig. 12 can be explained by the singlet state excimer formation time becoming smaller than the $90 \mathrm{~ns}$ fast signal integration window.

The use of sensors with different spectral sensitivity allows to study the second and the third continuum emis- 


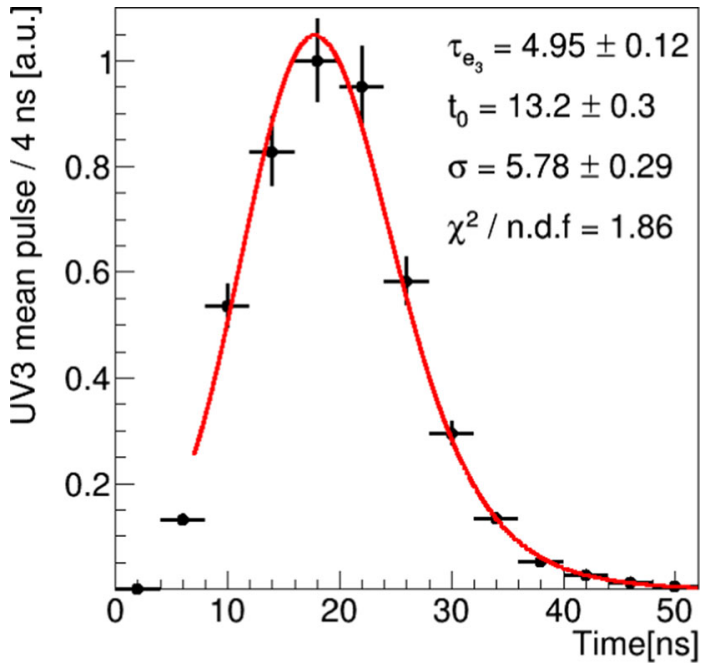

Fig. 15 Result of the fit (red line) of the mean pulse for $2 \times 10^{4} \alpha$ interactions in argon at 1.5 bar in the UV3 region (black) using Eq. 20

sions separately. The average light pulse (from $2 \times 10^{4}$ events) in the UV3 region with argon at 1.5 bar is shown in Fig. 15. Data are fitted using the following expression:

$I_{U V 3}(t)=\frac{L}{\tau_{f_{3}}-\tau_{e_{3}}}\left(e^{-t / \tau_{e_{3}}}-e^{-t / \tau_{f_{3}}}\right) \otimes G\left(t-t_{0}, \sigma\right)$,

where $L$ is a normalization constant, $G$ a gaussian function, with mean $t_{0}$ and width $\sigma$, that accounts for the detector response and $\tau_{f_{3}}$, and $\tau_{e_{3}}$ are the times necessary for the formation of the third continuum precursors and their characteristic photon emission time, respectively. In each bin of Fig. 15 the pulse error is calculated through the statistical distribution of the set of waveforms and a fixed uncertainty of $\pm 2 \mathrm{~ns}$ is introduced by the sampling rate of the ADC.

The $\tau_{f_{3}}$ time constant is fast and it cannot be precisely measured from the fit due to the $250 \mathrm{MHz}$ maximum sampling rate of our ADC, thus a limit $\tau_{f_{3}} \lesssim 1 \mathrm{~ns}$ is set on the molecular-ions formation time. The photon emission time constant $\tau_{e_{3}}$ is independent of the gas pressure in the range $[1,16]$ bar (Fig. 16) and its value is $5.02 \pm 0.11 \mathrm{~ns}$, calculated as the average of the fit results in the pressure range of interest. This result is compatible with previous works [24,31], where values of $\approx 5$ ns have been obtained for the lifetime of these radiative molecular states.

The time constants of the argon second continuum in the UV2 region are obtained in the $[1.5,8]$ bar pressure range, fitting the average light pulse in the UV2 region with the

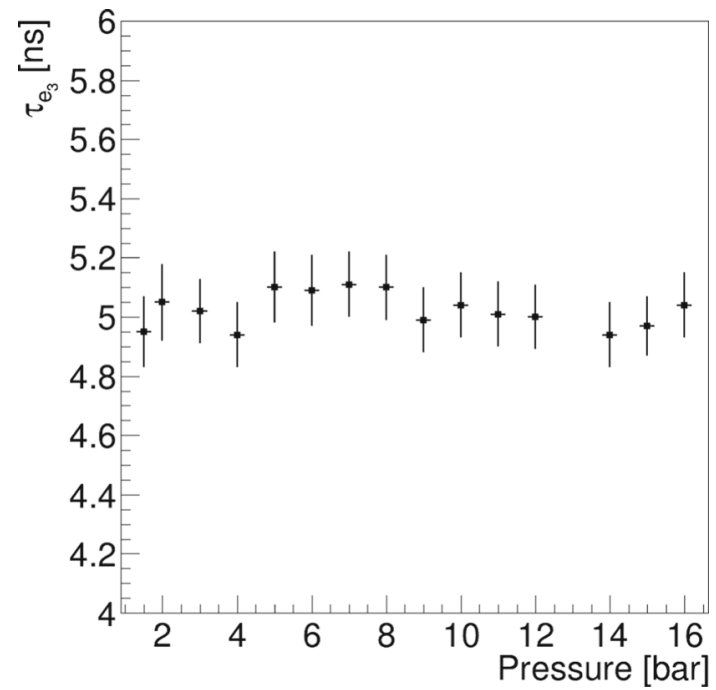

Fig. 16 Emission time constants calculated from the fit with Eq. 19 vs argon pressure

following function [40]:

$$
\begin{aligned}
I_{U V 2}(t)= & {\left[\frac{L_{1}}{\tau_{f_{2}}-\tau_{e_{2}}^{s}}\left(e^{-t / \tau_{f_{2}}}-e^{-t / \tau_{e_{2}}^{s}}\right)\right.} \\
& \left.+\frac{L_{2}}{\tau_{f_{2}}-\tau_{e_{2}}^{t}}\left(e^{-t / \tau_{f_{2}}}-e^{-t / \tau_{e_{2}}^{t}}\right)\right] \otimes G\left(t-t_{0}, \sigma\right),
\end{aligned}
$$

The two exponential differences account for the singlet $(s)$ and triplet $(t)$ contributions, independently, with the corresponding $\tau_{e_{2}}$ parameters labeled accordingly. The formation times have been assumed to be identical for both contributions. The result of the fit of the UV2-PMT average charge spectrum at 1.5 bar (Fig. 17-left) proves that Eq. 20 represents an accurate description of the argon scintillation signal at that pressure.

At pressures larger than 8 bar, the UV2 signal shape is characterized by a new structure a few $\mu$ s after the maximum pulse height, and Eq. 20 no longer describes the spectrum. A tentative explanation of this feature is taken from the model proposed in [42], that addresses the electron-ion recombination luminescence in the absence of electric field. This model considers a region of uniform ionization density and neglects the diffusion process out of the $\alpha$ track for the thermalized electrons. The recombination time $\tau_{r}$ is proportional to $P^{-2.7 \pm 0.3}$ for pressures larger than 8 bar [25] and it becomes shorter at larger pressures. In liquid argon, this time is in the ns range, where the time dependence of the recombination is dominated by the molecular de-excitation time.

A new term is added to Eq. 20 in order to describe the scintillation from the charge recombination, $I_{U V 2}^{R}$, which depends on the characteristic recombination time, $t_{r}$, and the excimer 
Fig. 17 Results of the fits (red lines) of the mean pulses for $2 \times 10^{4} \alpha$ interactions in argon at (left) 1.5 bar and (right) 16 bar, in the UV2 region (black dots). A 4 ns binning is used up to $100 \mathrm{~ns}$ and it is increased to $160 \mathrm{~ns}$ for larger times. Eq. 20 is used for the fit on the left, and Eq. $20+$ Eq. 21 for the fit on the right
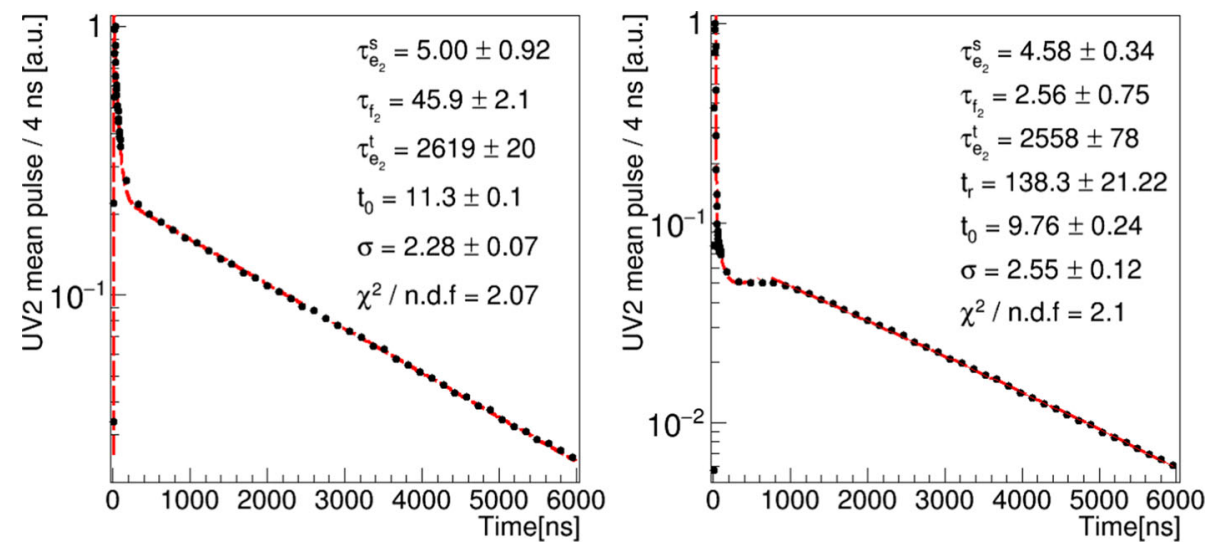

lifetime, $\tau_{e}$, (discussed in Sect. 2):

$$
\begin{array}{ll}
I_{U V 2}^{R}(t)=L_{3}\left(e^{-t / \tau_{e}^{t}}-e^{-2 t / t_{r}}\right), & \text { for } t<t_{r} \\
I_{U V 2}^{R}(t)=L_{3} e^{-t / \tau_{e}^{t}}, & \text { for } t>t_{r}
\end{array}
$$

where $L_{3}$ is a normalization constant. The result of the UV2 signal fit to Eq. 20, after including the additional term $I_{U V 2}^{R}(t)$, is shown in Fig. 17-right. The signal is successfully described by the fit above 8 bar up to 16 bar. The dependence of the excimer formation time and the singlet decay time in the UV2 region are summarized in Fig. 18 for different pressures.

The excimer formation time has a strong pressure dependence, decreasing from $46 \mathrm{~ns}$ at 1.5 bar to $2.6 \mathrm{~ns}$ at 16 bar. On the other hand, the singlet decay time emission is independent of the pressure with values around 4-5 ns. The triplet lifetime is measured to be $\approx 3 \mu$ s depending on the gas flow as expected. These results are in good agreement with previ-

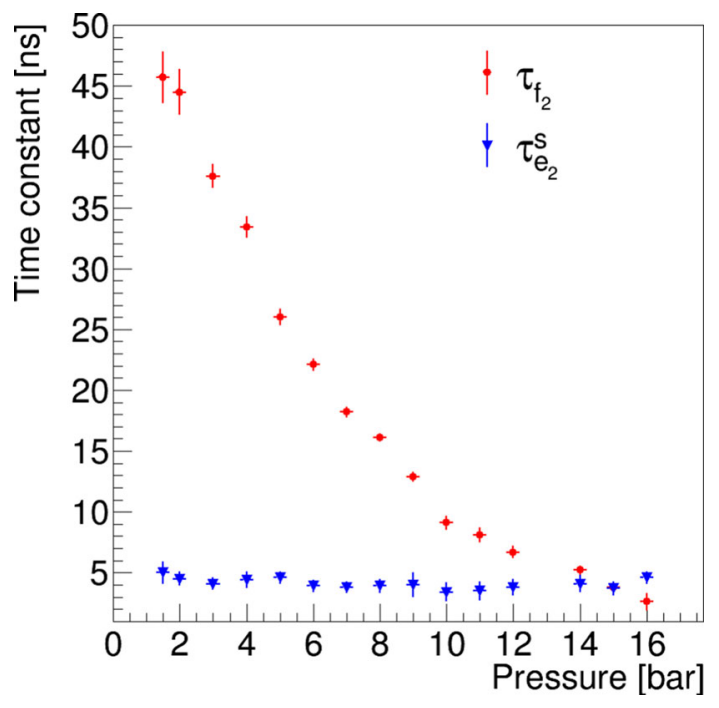

Fig. 18 Excimer formation time constant (red dots) and singlet deexcitation lifetime (blue triangles) vs argon pressure in the $1.5-16$ bar range ous studies [41]. At argon pressures below 5 bar, the excimer formation time is the dominant factor that determines the photon emission during the first hundred of ns. In this case, the singlet component of the second continuum emission is smeared over tens of ns. At higher gas pressure, the typical excimer formation time decreases.

\section{Electric field dependence of the scintillation and spectroscopic studies of the electron-ion recombination}

With the aim of establishing the nature of the new structure that appears in the UV2 range at large pressures and to study the field dependence of the UV3 emission, a small field cage ( $2 \mathrm{~cm}$ height) with the ${ }^{241} \mathrm{Am}$ source on the anode plate was introduced in the central volume of the pressure chamber.

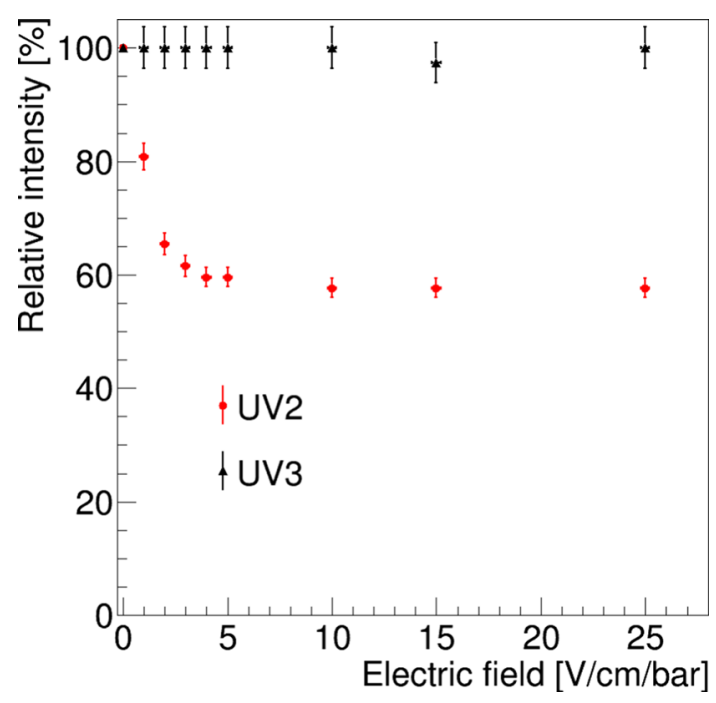

Fig. 19 Variation of the luminescence with the electric field intensity in the UV2 (red dots) and UV3 (black triangles) regions. The UV3 signal is not affected by the electric field 
Fig. 20 Mean pulse shape of the UV2 (left) and UV3 (right) signals with (blue line) and without (black line) electric field with argon at 10 bar. The bump in the UV2 graph disappears when the electric field is applied. The UV3 signal is not affected by the electric field
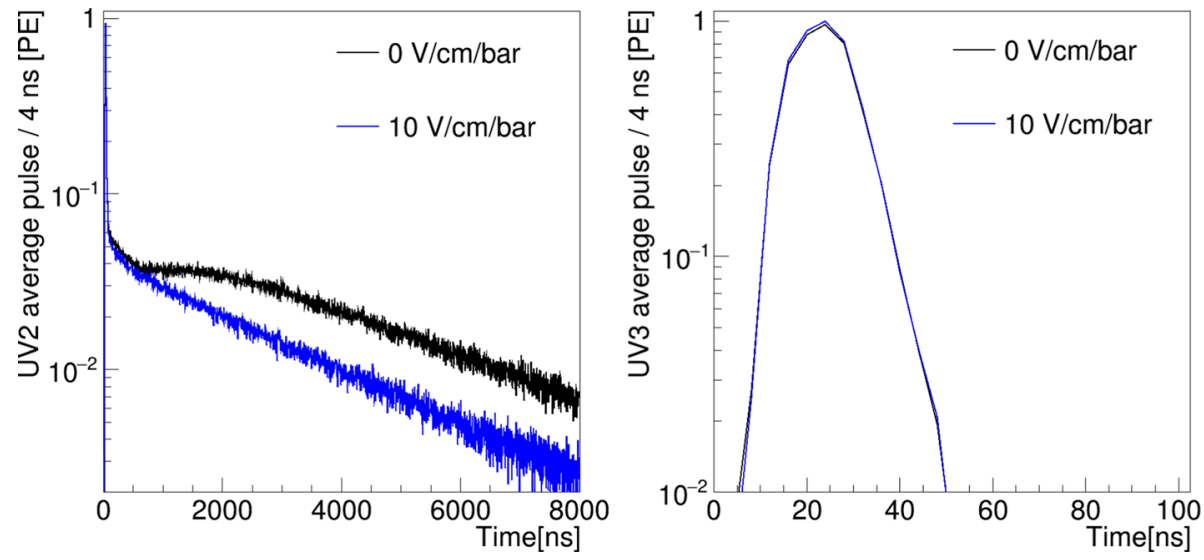

Data at 10 bar of pressure were taken in order to fully contain the $\alpha$ track in the field region. In this setup the anode is grounded and the reduced electric field $(\mathrm{E} / \mathrm{P})$ applied in the range from 0 to $25 \mathrm{~V} / \mathrm{cm} / \mathrm{bar}$. The number of PE measured with the ${ }^{241} \mathrm{Am}$ peak as a function of the electric field is shown in Fig. 19, evidencing that the emission in the UV2 wavelength range decreases with electric fields up to $4 \mathrm{~V} / \mathrm{cm} / \mathrm{bar}$ and then remains constant. The saturation in the collection of the charge above this field value is consistent with the measurements reported in [25] for the second continuum. On the other hand, the emission in the UV3 spectral region is not affected by the electric field up to $25 \mathrm{~V} / \mathrm{cm} / \mathrm{bar}$.

The scintillation pulses at 10 bar with and without a $10 \mathrm{~V} / \mathrm{cm} / \mathrm{bar}$ reduced field, averaged over $2 \times 10^{4}$ events and normalized to the maximum value of the distribution, are shown in Fig. 20, both for the UV2 and UV3 spectral ranges. The bump above $1 \mu$ s in the UV2 range (left graph) disappears by increasing the amplitude of the reduced field. This result confirms the interpretation of this structure in terms of electron-ion recombination given in Sect. 5. When a sufficiently strong field is applied, the charge recombination is suppressed and the overall signal shape is similar to the scintillation pulse at pressures below 8 bar. In these conditions, the argon slow component is well described by a single exponential function.

The distribution for UV3 (right plot) proves that the photon emission in this spectral region is not affected by the electric field. This result demonstrates that the recombination light is consistent with the second continuum emission at $128 \mathrm{~nm}$, only.

\section{First evidence of the third continuum emission with a bupbeta source and scope of this project}

Our experiments with $\alpha$ particles in argon at different pressures have proved that a substantial component of the scintillation is in the range $[160,325] \mathrm{nm}$ and is not related to the radiative de-excitation of the singlet and triplet excimers.

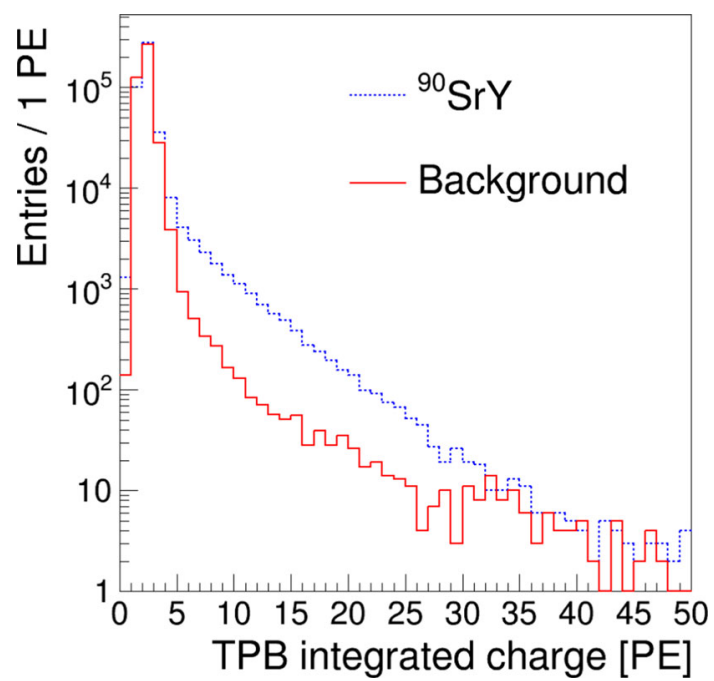

Fig. 21 Integrated charge of the TPB-PMT for events collected without (red line) and with (blue line) the ${ }^{90} \mathrm{Sr} / Y \beta$ source in the center of the detector. The number of events is normalized to the same acquisition time

We interpret this photon production through the mechanism responsible for the third continuum emission (Sect.2). Additional studies have been performed replacing the ${ }^{241} \mathrm{Am} \alpha$ source with a weak ${ }^{90} \mathrm{Sr} / Y \beta$ source (activity $\approx 100 \mathrm{~Bq}$ ), with $546 \mathrm{keV}$ and $2280 \mathrm{keV}$ Q-values of the decays. We collected several runs with argon in the pressure range $[15,21]$ bar. The $\beta$ 's from the source are typically not fully contained in our detector, unlike for the case of $\alpha$ interactions. To increase the light collection, the UV2-PMT (R6835) is replaced by another phototube coated with TPB (R6095). In this configuration, the trigger is produced by the coincidence of the two TPB-PMTs.

The integrated charge spectrum of one TPB-PMT, obtained with the ${ }^{90} \mathrm{Sr} / Y \beta$ source and gas argon at 20 bar is compared with a background spectrum taken without any source (Fig. 21). A prominent excess is present at low energy, between 6 and $25 \mathrm{PE}$, giving us a solid evidence of the actual identification of the $\beta$ interactions. A typical signal produced by one UV-PMT and one TPB-PMT in this region is dis- 

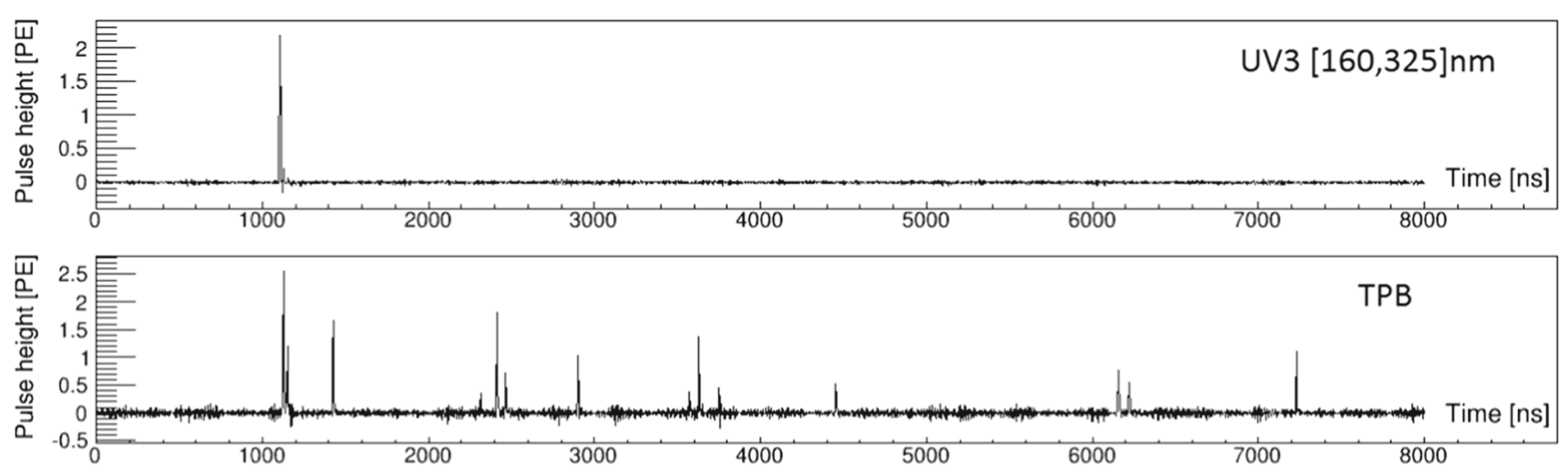

Fig. 22 Typical Ar scintillation signals detected for a $\beta$ event in two spectral regions, with argon gas at 20 bar

played in Fig. 22. A clear pulse, similar to the one detected with the $\alpha$ source but with an amplitude consistent with the different energy scale, is shown in the UV3 region in coincidence with the TPB-PMT signal. This result is a first evidence for a possible third continuous emission produced by $\beta$ particles from radioactive sources in argon gas. It should be noted that spurious effects in the UV3 region (interactions with walls or windows) cannot be fully excluded since, unlike $\alpha$ interactions, $\beta$ 's are typically not completely contained in the chamber. Further studies with the ${ }^{90} \mathrm{Sr} / \mathrm{Y}$ source are currently underway.

This work demonstrates, on the one hand, the presence of copious scintillation yields of third continuum emission from $\alpha$ and $\beta$ interactions in argon gas, while, on the other hand, it conveys evidence that the temporal and spectroscopic information of the argon scintillation are strongly correlated, and can be used independently or complementary for particle identification. New studies on this front are currently ongoing [43]. Additionally, similar observations in xenon would trigger immediate interest for xenon-based detectors and are currently being explored. Also interestingly, the ratio of the $3 \mathrm{rd} / 2 \mathrm{nd}$ continuum determined here is $0.20 \pm 0.14$ at 2 bar (where recombination is small) and is $0.18 \pm 0.12$ at 10 bar, with a drift fields of $25 \mathrm{~V} / \mathrm{cm} / \mathrm{bar}$. If these yields can be maintained in the presence of a suitably chosen VUV-quenching and/or VUV-absorbing additive, 3rd continuum scintillation would offer a plausible source of primary scintillation for gas detectors read through avalanche multiplication. Since this emission will result in a much lower photoelectric effect yield compared to the 2 nd continuum component, it might be possible to obtain good avalanche gain for a gaseous detector operated in its presence. In particular, this could be advantageous for high-pressure argon time projection chambers such as the one foreseen at the Near Detector Complex of the DUNE experiment, which is currently planned to be read out through avalanche multiplication [9].

\section{Conclusions}

Conventional dark matter and neutrino detectors based on noble element time-projection chambers are built with fast light detection devices coupled with photon wavelengthshifters that integrate the light signal over a wide spectral range, missing the potential information provided by the scintillation wavelength.

We have studied the primary and the recombination scintillation of the argon gas with a wavelength sensitive detector operated with $\alpha$ and $\beta$ sources electrodeposited on stainless steel disks. Our results evidence the emission of photons at wavelengths larger than the $128 \mathrm{~nm}$ line through a production mechanism which is not based on the excimer formation associated to the low-lying atomic states. We interpret this component of the argon scintillation as the third continuum emission.

We have proven that up to $20 \%$ of the scintillation obtained with an ${ }^{241} \mathrm{Am}$ source in 1.5 bar argon gas is in the range $[160,325] \mathrm{nm}$. The photon yield and the typical emission time of this component are largely independent on the gas pressure up to 16 bar, and the emission is not significantly affected by an external electric field. Compared to the second continuum, which is dominated by the excimer formation time, the third continuum is remarkably fast $\left(\tau_{e_{3}}=5.02 \pm 0.11 \mathrm{~ns}\right)$ and represents the main contribution to the argon light signal during the first tens of ns, for pressures below 10 bar. The spectroscopic studies of the electron-ion recombination light revealed that this component is consistent with the $128 \mathrm{~nm}$ emission. Evidence of the third continuum emission produced by $\beta$ interactions in argon gas has been also obtained using a ${ }^{90} \mathrm{Sr} / \mathrm{Y}$ source.

We have established that the argon second and third continuum scintillation can be distinguished experimentally by means of sensors with different spectral sensitivities, making possible to exploit distinctive features of the noble gases photon emission that are not envisaged by the present experiments. Particularly, our investigations open new paths toward 
a novel particle identification technique based on the spectral information of the noble-elements scintillation light. Work to extend this study to Xe gas and liquid Ar is ongoing.

Acknowledgements This research is funded by the Spanish Ministry of Economy and Competitiveness (MINECO) through the grant FPA2017-92505-EXP. The authors are also supported by the "Física de partículas" Unit of CIEMAT through the grant MDM-2015-0509. DGD is supported by the Ramon y Cajal program (Spain) under contract number RYC-2015-18820.

Data Availability Statement This manuscript has associated data in a data repository. [Authors' comment: The datasets analysed during the current study are available from the corresponding author on reasonable request.]

Open Access This article is licensed under a Creative Commons Attribution 4.0 International License, which permits use, sharing, adaptation, distribution and reproduction in any medium or format, as long as you give appropriate credit to the original author(s) and the source, provide a link to the Creative Commons licence, and indicate if changes were made. The images or other third party material in this article are included in the article's Creative Commons licence, unless indicated otherwise in a credit line to the material. If material is not included in the article's Creative Commons licence and your intended use is not permitted by statutory regulation or exceeds the permitted use, you will need to obtain permission directly from the copyright holder. To view a copy of this licence, visit http://creativecomm ons.org/licenses/by/4.0/.

Funded by SCOAP ${ }^{3}$.

\section{References}

1. DarkSide collaboration, P. Agnes et al., Low-mass dark matter search with the DarkSide-50 experiment. Phys. Rev. Lett. 121(8), 081307 (2018). https://doi.org/10.1103/PhysRevLett.121.081307

2. ArDM collaboration, J. Calvo et al., Commissioning of the ArDM experiment at the Canfranc underground laboratory: first steps towards a tonne-scale liquid argon time projection chamber for Dark Matter searches. JCAP 03, 003 (2017). https://doi.org/10. 1088/1475-7516/2017/03/003

3. DEAP collaboration, R. Ajaj et al., Search for dark matter with a 231-day exposure of liquid argon using DEAP-3600 at SNOLAB. Phys. Rev. D 100(2), 022004 (2019). https://doi.org/10. 1103/PhysRevD.100.022004

4. XENON collaboration, E. Aprile et al., Dark matter search results from a one ton-year exposure of XENON1T. Phys. Rev. Lett. 121(11), 111302 (2018). https://doi.org/10.1103/PhysRevLett. 121.111302

5. DarkSide collaboration, C.E. Aalseth et al., DarkSide-20k: A 20 tonne two-phase LAr TPC for direct dark matter detection at LNGS. Eur. Phys. J. Plus 133, 131 (2018). https://doi.org/10.1140/epjp/ i2018-11973-4

6. EXO-200 collaboration, J. Albert et al., Search for Majorana neutrinos with the first two years of EXO-200 data. Nature 510, 229-234 (2014). https://doi.org/10.1038/nature13432

7. MicroBooNE collaboration, R. Acciarri et al., Design and construction of the MicroBooNE Detector. JINST 12(02), P02017 (2017). https://doi.org/10.1088/1748-0221/12/02/P02017

8. DUNE collaboration, Abi et al., First results on ProtoDUNE-SP liquid argon time projection chamber performance from a beam test at the CERN Neutrino Platform. JINST 15(12), P12004 (2020). https://doi.org/10.1088/1748-0221/15/12/P12004
9. DUNE collaboration, High-Pressure Argon gas TPC Option for the DUNE Near Detector (2021). arXiv:1910.06422 [physics.ins-det]. https://docs.dunescience.org/cgi-bin/RetrieveFile?docid=12388\& filename=High_Pressure_Argon_gas_TPC_Option_for_the DUNE_Near_Detector.pdf\&version $=1$

10. NEXT collaboration, V. Alvarez et al., NEXT-100 Technical Design Report (TDR): executive summary. JINST 7, T06001 (2012). https://doi.org/10.1088/1748-0221/7/06/T06001

11. DEAP collaboration, P. Adhikari et al., The liquid-argon scintillation pulseshape in DEAP-3600. Eur. Phys. J. C 80(4), 303 (2020). https://doi.org/10.1140/epjc/s10052-020-7789-x

12. ArDM collaboration, J. Calvo et al., Backgrounds and pulse shape discrimination in the ArDM liquid argon TPC. JCAP 12, 011 (2018). https://doi.org/10.1088/1475-7516/2018/12/011

13. M.V. McCusker, in Excimer Lasers, Topics in Applied Physics, 2nd edn., edited by Ch. K. Rhodes, Chapt. 3, vol. 30 (Springer, Berlin, 1984)

14. C.M.B. Monteiro, J.A.M. Lopes, J.F.C.A. Veloso, J.M.F. dos Santos, Secondary scintillation yield in pure argon. Phys. Lett. B 668, 167-170 (2008). https://www.sciencedirect.com/science/ article/pii/S0370269308010435

15. E. Robert, A. Khacef, C. Cachoncinlle, J.M. Pouvesle, Timeresolved spectroscopy of high pressure rare gases excited by an energetic flash X-ray source. Opt. Commun. 117, 179 (1995). https://doi.org/10.1016/0030-4018(94)00664-G

16. H.A. Koehler, L.J. Ferderber, D.L. Redhead, P.J. Ebert, Vacuumultraviolet emission from high-pressure xenon and argon excited by high-current relativistic electron beams. Phys. Rev. A 9, 768 (1974). https://doi.org/10.1103/PhysRevA.9.768

17. R. Sauerbrey, F. Emmert, H. Langhoff, Fluorescence and absorption in electron beam excited argon. J. Phys. B 17, 2057 (1984). https://iopscience.iop.org/article/10.1088/0022-3700/17/10/011

18. T. Heindl, T. Dandl, M. Hofmann, R. Krucken, L. Oberauer, W. Potzel, J. Wieser, A. Ulrich, The scintillation of liquid argon. EPL 91, 62002 (2010). https://iopscience.iop.org/article/10.1209/ 0295-5075/91/62002/pdf

19. G.S. Hurst, T.E. Bortner, T.D. Strickler, Proton excitation of the argon atom. Phys. Rev. 178, 4 (1969). https://doi.org/10.1103/ PhysRev.178.4

20. A. Ulrich et al., Heavy ion excitation of rare-gas excimers. J. Appl. Phys. 62, 357 (1987). https://doi.org/10.1063/1.339804

21. J. Wieser, A. Ulrich, A. Fedenev, M. Salvermoser, Novel pathways to the assignment of the third rare gas excimer continua. Opt. Commun. 173, 233 (2000). https://doi.org/10.1016/ S0030-4018(99)00610-0

22. C.D.R. Azevedo, D. González-Díaz, S.F. Biagi, C.A.B. Oliveira, Microscopic simulation of xenon-based optical TPCs in the presence of molecular additives. Nucl. Instrum. Methods A 877, 157 (2018). https://doi.org/10.1016/j.nima.2017.08.049

23. G. Klein, M.J. Carvalho, Argon luminescence bands between 1600 and 2900 Å. J. Phys. B At. Mol. Phys. 14, 1283 (1981). https:// iopscience.iop.org/article/10.1088/0022-3700/14/8/013

24. A. Birot, H. Brunet, J. Galy, P. Millet, J.L. Teyssier, Continuous emissions of argon and krypton in the near ultraviolet. J. Chem. Phys. 63, 1469 (1975). https://doi.org/10.1063/1.431509

25. M. Suzuki, J. Ruan, S. Kubota, Time dependence of the recombination luminescence from high-pressure argon, krypton, and xenon excited by alpha particles. Nucl. Instrum. Methods 192, 565 (1982). https://doi.org/10.1016/0029-554X(82)90874-6

26. V. Aquilanti, S. Cavalli, S.G. Grossi, Hund's cases for rotating diatomic molecules and for atomic collisions: angular momentum coupling schemes and orbital alignment. Z Phys. D At. Mol. Clust. 36, 215-219 (1996). https://doi.org/10.1007/BF01426406

27. T. Robert Connor, M. Biondi, Dissociative recombination in neon: spectral line-shape studies. Phys. Rev. 150, A778 (1965). https:// doi.org/10.1103/PhysRev.140.A778 
28. L. Frommhold, M.A. Biondi, Interferometric study of dissociative recombination radiation in neon and argon afterglows. Phys. Rev. 185, 244 (1969). https://doi.org/10.1103/PhysRev.185.244

29. M. Suzuki, J. Ruan, S. Kubota, Electric field dependence of the decay curves of high pressure xenon scintillators excited by alpha particles. Nucl. Instrum. Methods 192, 623 (1982). https://doi.org/ 10.1016/0029-554X(82)90884-9

30. K. Saito, H. Tawara, T. Sanami, E. Shibamura, S. Sasaki, Absolute number of scintillation photons emitted by alpha particles in rare gases. IEEE Trans. Nucl. Sci. 49, 4 (2002). https://ieeexplore.ieee. org/document/1043429

31. H. Langhoff, The origin of the third continua emitted by excited rare gases. Opt. Commun. 68, 1 (1988). https://doi.org/10.1016/ 0030-4018(88)90008-9

32. A. Boichenko, V. Tarasenko, S. Yakovlenko, Nature of third continua in rare gases, in Proc. SPIE., International Conference on Atomic and Molecular Pulsed Lasers III, vol. 4071. https://doi. org/10.1117/12.383464

33. C. Benson, G.D. Orebi Gann, V. Gehman, Measurement of the intrinsic quantum efficiency and visible reemission spectrum of tetraphenyl butadiene thin films for incident vacuum ultraviolet light. Eur. Phys. J. C 78, 329 (2018). https://doi.org/10.1140/epjc/ s10052-018-5807-z

34. https://www.hamamatsu.com/resources/pdf/etd/R7378A_ TPMH1288E.pdf

35. https://www.hamamatsu.com/resources/pdf/etd/R6835_ TPMH1263E.pdf

36. C. Benson, G. Orebi Gann, V. Gehman, Measurements of the intrinsic quantum efficiency and absorption length of tetraphenyl butadiene thin films in the vacuum ultraviolet regime. Eur. Phys. J. C 78(4), 329 (2018). https://link.springer.com/article/10.1140/epjc/ s10052-018-5807-z1
37. https://www.thorlabs.com/NewGroupPage9.cfm?ObjectGroup $\mathrm{ID}=3982$

38. https://www.asahi-spectra.com/opticalfilters/detail.asp? $\mathrm{key}=$ XUL0250

39. M. Akashi-Ronquest et al., Triplet lifetime in gaseous argon. Eur. Phys. J. A 55(10), 176 (2019). https://doi.org/10.1140/epja/ i2019-12867-2

40. C. Amsler, V. Boccone, A. Buchler, R. Chandrasekharan, C. Regenfus, J. Rochet, Luminescence quenching of the triplet excimer state by air traces in gaseous argon. JINST 3, P02001 (2008). https:// doi.org/10.1088/1748-0221/3/02/P02001

41. J.W. Keto, R.E. Gleason Jr., G.K. Walters, Production mechanisms and radiative lifetime of argon and xenon molecules emitting in the ultraviolet. Phys. Rev. Lett. 33, 1365 (1974). https://doi.org/ 10.1103/PhysRevLett.33.1365

42. S. Kubota, M. Hishida, M. Suzuki, J. Ruan, Dynamical behaviour of free electrons in the recombination process in liquid argon, krypton and xenon. Phys. Rev. B 20, 3486 (1979). https://doi.org/10.1103/ PhysRevB.20.3486

43. R. Santorelli, E. Sanchez, Particle identification with the argon scintillation spectroscopy (2021) (paper in preparation) 